\title{
Diversidad de gorgojo de los Andes (Coleoptera: Curculionidae) en papa (Solanum spp.) en Puno-Perú
}

\author{
Diversity of Andean potato weevils (Coleoptera: Curculionidae) \\ on Potato (Solanum spp.) in Puno-Peru
}

\section{(iD ROSARIO Y.BRAVO-PORTOCARRERO ${ }^{1}$; (D) VÍCTOR HUGO IDME- BUSTINZA $^{1}$; (iD ISRAEL LIMA-MEDINA ${ }^{1}$ (ID GROVER CORNEJO-CONDORI ${ }^{1}$; (iD VÍCTOR HUGO CASA-COILA ${ }^{2}$}

\footnotetext{
${ }^{1}$ Universidad Nacional del Altiplano, Puno, Perú, rosariobravo@unap.edu.pe,idbus@hotmail.com,ilima@unap.edu.pe,grovercornejo7@gmail.com ${ }^{2}$ Universidad Nacional San Agustín de Arequipa, vcasac@unsa.edu.pe
}

\begin{abstract}
*Autor para correspondencia
Rosario Y. Bravo-Portocarrero, Universidad Nacional del Altiplano-Puno-Perú, Ciudad Universitaria, Av. Floral No 1153 , Código postal 21001,rosariobravo@unap.edu.pe, https://orcid.org/0000-0001-5186-7569

\section{Citación sugerida}

BRAVO-PORTOCARRERO, R. Y.; IDME-BUSTINZA, V. H.; LIMA-MEDINA, I.; CORNEJO-CONDORI, G.; CASA-COILA, V. H. 2021. Diversidad de gorgojo de los Andes (Coleoptera: Curculionidae) en papa (Solanum spp.) en Puno-Perú. Revista Colombiana de Entomología 47 (2): e10797. https://doi. org/10.25100/socolen.v47i2.10797

Recibido: 05-Ene-2021

Aceptado: 31-Ago-2021

Publicado: 12-Nov-2021

Revista Colombiana de Entomología ISSN (Print): 0120-0488

ISSN (On Line): 2665-4385

https://revistacolombianaentomologia.univalle.edu.co

Open access

(c) (i) (2) (2) BY-NC-SA 4.0
\end{abstract}

Publishers: Sociedad Colombiana de Entomología SOCOLEN (Bogotá, D. C., Colombia) https://www.socolen.org.co

Universidad del Valle (Cali, Colombia)

https://www.univalle.edu.co

(C) 2021 Sociedad Colombiana de Entomología SOCOLEN y Universidad del Valle - Univalle
Resumen: El Altiplano peruano es considerado como lugar de origen del cultivo de papa (Solanum spp.), así mismo, sería el centro de origen de sus plagas insectiles más importantes compuesta por un conjunto de diferentes especies denominado complejo gorgojo de los Andes (Coleoptera: Curculionidae), siendo el objetivo del trabajo estudiar la diversidad de especies que conforman el complejo de estas plagas, en el ámbito de dos comunidades productoras de papa: Jallahua y Kcoriñahui, distantes entre sí $17 \mathrm{~km}$ y ubicadas a altitudes de 3883 y 4093 msnm, en el distrito de Pucará (Lampa-Puno). Para ello se colectaron adultos de gorgojos plaga, con trampas de caída Barber modificadas; luego se diferenciaron e identificaron por comparación con material referencial de la colección del Centro Internacional de la papa CIP y posteriormente ratificada su identificación mediante el uso de claves dicotómicas. Como resultado fue posible asignar los ejemplares a las siguientes especies: Premnotrypes solaniperda Kuschel, Cylydrorhinus sp., Listroderes punicola Kuschel, Puranius sp., Rigopsidius tucumanus Heller, Rigopsidius piercei Heller y cuatro especies aún no identificadas taxonómicamente; lo cual sugiere un alto nivel de diversidad aún en áreas geográficas cercanas. Se encontró además heterogeneidad en la distribución geográfica de las especies, siendo altamente dominante $P$. solaniperda, en tanto que el resto de las especies, muestran densidades e índices de dominancia intermedios y bajos.

Palabras clave: Premnotrypes, Rigopsidius, Solanum tuberosum, Solanum spp.

Abstract: The Peruvian Altiplano is considered the place of origin of the potato crop (Solanum spp.). The Altiplano is also the center of the origin of the most important insect pests, compound by different species, called the Andean potato weevils complex (Coleoptera: Curculionidae). The present study aimed to demonstrate the diversity of species of the pests in two potato-producing communities: Jallahua and Kcoriñahui, distant between them $17 \mathrm{~km}$ and located at altitudes between 3883 and 4093 meters above sea level (a.s.1.), in the district Pucará, Lampa, Puno. Adults of the Andean weevil were collected from potatoes plants with Barber's pitfall traps, then they were classified and identified by comparison with reference material from the collection of the International Potato Center (CIP) and subsequently the identifications were verified in the laboratory with a dichotomous key. Results allowed differentiating the species: Premnotrypes solaniperda Kuschel, Cylydrorhinus sp., Listroderes punicola Kuschel, Puranius sp., Rigopsidius tucumanus Heller, Rigopsidius piercei Heller, and four unidentified species. Heterogeneity in the geographical distribution of the species was verified with Premnotrypes solaniperda Kuschel being the most dominant, the other species showed intermediate and lower densities and dominances.

Keywords: Premnotrypes, Rigopsidius, Solanum tuberosum, Solanum spp.

\section{Introducción}

El cultivo de papa es uno de los de mayor importancia en la alimentación y soporte económico de la población altiplánica Cahuana y Arcos (2002). Los productores afrontan un problema persistente, causado por varias especies de gorgojos plaga de mayor importancia para el cultivo en esta región altiplánica. Este complejo se encuentra conformado por coleópteros de la familia Curculionidae, pertenecientes a los géneros Premnotrypes, Rhygopsidius y otros relacionados (Alcázar 2002). 
Con una distribución geográfica de especies del complejo gorgojo de los Andes, desde Venezuela hasta Argentina, en todas las zonas productoras de papa de los Andes americanos (Alcázar et al. 2016). Las larvas de los gorgojos producen daños de importancia económica directamente a los tubérculos, mientras que los daños causados por los gorgojos adultos son esporádicos y de menor gravedad, usualmente en las hojas basales de las plantas (Bravo 2010; Zapana et al. 2015; Loza y Bravo 2015).

Las zonas agroecológicas Circunlacustre y Suni de la Región Puno, son las de mayor producción de diversos cultivares dulces y amargos de papa. Son también las zonas donde se encuentra mayor diversidad de especies de gorgojo de los Andes (Cahuana y Arcos 2002). Así en la región Puno, se han registrado las siguientes especies sobre papa: Premnotrypes solaniperda Kuschel, 1956, Rhigopsidius tucumanus Heller,1906, Rhigopsidius piercei Heller, 1936 Listroderes punicola Kuschel, 1949 (Coleoptera:Curculionidae) encontrándose además Mycrotrypes sp. en cultivo de oca (Oxalis tuberosa) (Bravo 2010). En Potosí se identificó R. tucumanus, como la especie más abundante y dañina en plantaciones de papa (Barea et al. 2016), ratificado por Jarandilla (2010) en la localidad de Aroma La Paz-Bolivia.

Los gorgojos de este complejo de insectos aún no han sido todos identificados a nivel de género $\mathrm{y} / \mathrm{o}$ especie ni estudiados para cada zona productora de papa (Kroschel et al. 2012). Yábar-Landa (2011) registró siete especies de curculiónidos asociados a tubérculos de papa en condiciones de almacenamiento tradicional en Cusco-Perú: Cylidrorhinus sp., Listroderes sp., Premnotrypes latithorax Pierce, 1914 (Coleoptera:Curculionidae) P. solaniperda, Premnotrypes suturicallus Kuschel, 1949 (Coleoptera: Curculionidae), Premnotrypes sp., Puranius obrienorum Morrone, 1994 (Coleoptera: Curculionidae) y Puranius sp.

Premnotrypes pusilus Kuschel, 1956, (Coleoptera: Curculionidae)es una especie recientemente reportada, luego de su descripción original y redescrita por Yábar-Landa (2015b) quien además amplió su distribución geográfica de los departamentos de Pasco, Junín y Cusco hacia el departamento de Apurímac, con altitudes de 3690 a 4400 msnm. Yábar-Landa (2019) reportó nuevos registros de tres especies de Microtrypes Kuschel, 1949 y nuevas localidades para Rhynotrypes Kuschel, 1956 (Curculionidae: Entiminae: Premnotrypine) en Perú. La distribución de la sub-familia Entiminae, se circunscribe a la Cordillera de los Andes, desde Colombia, hasta Bolivia y el norte de Chile, por lo general a alturas mayores a $3000 \mathrm{msnm}$.

La biodiversidad en su definición más simple es la variedad y variabilidad de los seres vivos, entendida como la riqueza o número de especies distintas en un área geográfica determinada. Los métodos para medir la diversidad de especies se basan en cuantificar el número de las que están presentes en términos de abundancia (Moreno 2002). Los índices de diversidad basados en la abundancia más utilizados en ecología son el de Shannon o el de Gini-Simpson, que son medidas relacionadas con la complejidad de las comunidades biológicas (Jost y Gonzales-Oreja 2012). Uno de los aspectos más importantes de la biodiversidad es que, cuando ésta es mayor permite enfrentar mejor los cambios que puedan producirse como consecuencia de las alteraciones en el clima, por efectos del cambio climático (Yábar et al. 2015a).

En los sistemas agrícolas, los seres humanos y los insectos han encontrado un espacio de interacción multifactorial como componentes, con niveles de diversidad en los agroecosistemas (Guzmán et al. 2016). Con respecto a la competencia Begón y Towsend (1995), manifestaron que ésta sólo puede ocurrir si un recurso se encuentra limitado, siendo posible la coexistencia, como lo informa Cabello et al. (1999) cuando el ambiente es heterogéneo, si existe dispersión y si las especies se encuentran agregadas espacialmente. A partir de la información sobre abundancia y diversidad de especies de gorgojo de los Andes, tanto en Puno como en otros lugares del país y América, se planteó como objetivo de esta investigación: estimar la diversidad de especies del complejo gorgojo de los Andes, en el ámbito de dos comunidades productoras de papa en el distrito de Pucara (Lampa- Puno), Perú.

\section{Materiales y métodos}

La captura de especímenes adultos de gorgojos de los Andes se realizó en las comunidades Jallahua, ubicada a $3883 \mathrm{msnm}$ (UTM 19L 350293.23E 8327120.44S) y Kcoriñahui a 4093 msnm (UTM 19L 344475.78E 8336911.945S), en el distrito de Pucará (Lampa - Puno - Perú), sobre un área geográfica de $42,79 \mathrm{~km}^{2}$ (Fig. 1). Ambas comunidades se encuentran localizadas en la zona agroecológica Suni de la región Puno y fueron seleccionadas al azar entre 29 del distrito de Pucará; en ellas se seleccionaron ocho parcelas de similares dimensiones con variedades nativas de papa.

Para las capturas de los gorgojos adultos se utilizaron trampas de caída Barber modificadas (Fig. 2 A, B) elaboradas a partir de botellas descartables, con $10 \mathrm{~cm}$ de diámetro y 20 de profundidad, cortadas cerca del cuello, con la parte superior en forma de embudo y una madera forrada con plástico como tapa. Se enterraron los envases con la apertura a nivel de la superficie del suelo y se dejó un espacio de tres centímetros para el ingreso de los gorgojos adultos. Las trampas de caída fueron colocadas antes de la emergencia de las plantas y distribuidas 10 en cada parcela. Las evaluaciones se realizaron cada quince días durante seis meses en la campaña agrícola 2013-2014, se recogieron los especímenes atrapados y registraron el número de individuos por trampa.

Las especies de gorgojos de los Andes colectados se montaron y se procedió luego a su identificación inicialmente por comparación con material de referencia de la colección del museo del Centro Internacional de la Papa (CIP), Lima-Perú. Posteriormente se corroboró en el laboratorio de entomología de la FCA-UNA-Puno. Para ello, se utilizaron claves de identificación taxonómica con base en caracteres morfológicos (Kuschel, 1949, 1956; Morrone y Loiacono 1992). Las observaciones, se realizaron con microscopio estereoscópico binocular marca Carl Zeiss, modelo Stemi DV4, sistema de iluminación LED y margen de aumento de 8 a $32 x$, extendible a $64 x$ con lente opcional $2 x$.

La diversidad y distribución de especies en ambas comunidades, se obtuvo en base al número de individuos colectados/especie/trampa/parcela de cada comunidad. Para calcular los índices de diversidad basados en la abundancia se usó la fórmula descrita por Jost y González-Oreja (2012), que combinan la riqueza de especies con su equitatividad. Esto lo explica cuantitativamente Giordanengo et al. (2013) como una medida de dominancia expresada como: $\mathrm{D}=1-3 \mathrm{p}^{2}$. Siendo $\mathrm{p}^{2}=\mathrm{n}_{\mathfrak{i}} / N$, es la abundancia total de las especies e interpretando que, a menores índices mayor diversidad o dominancia. 


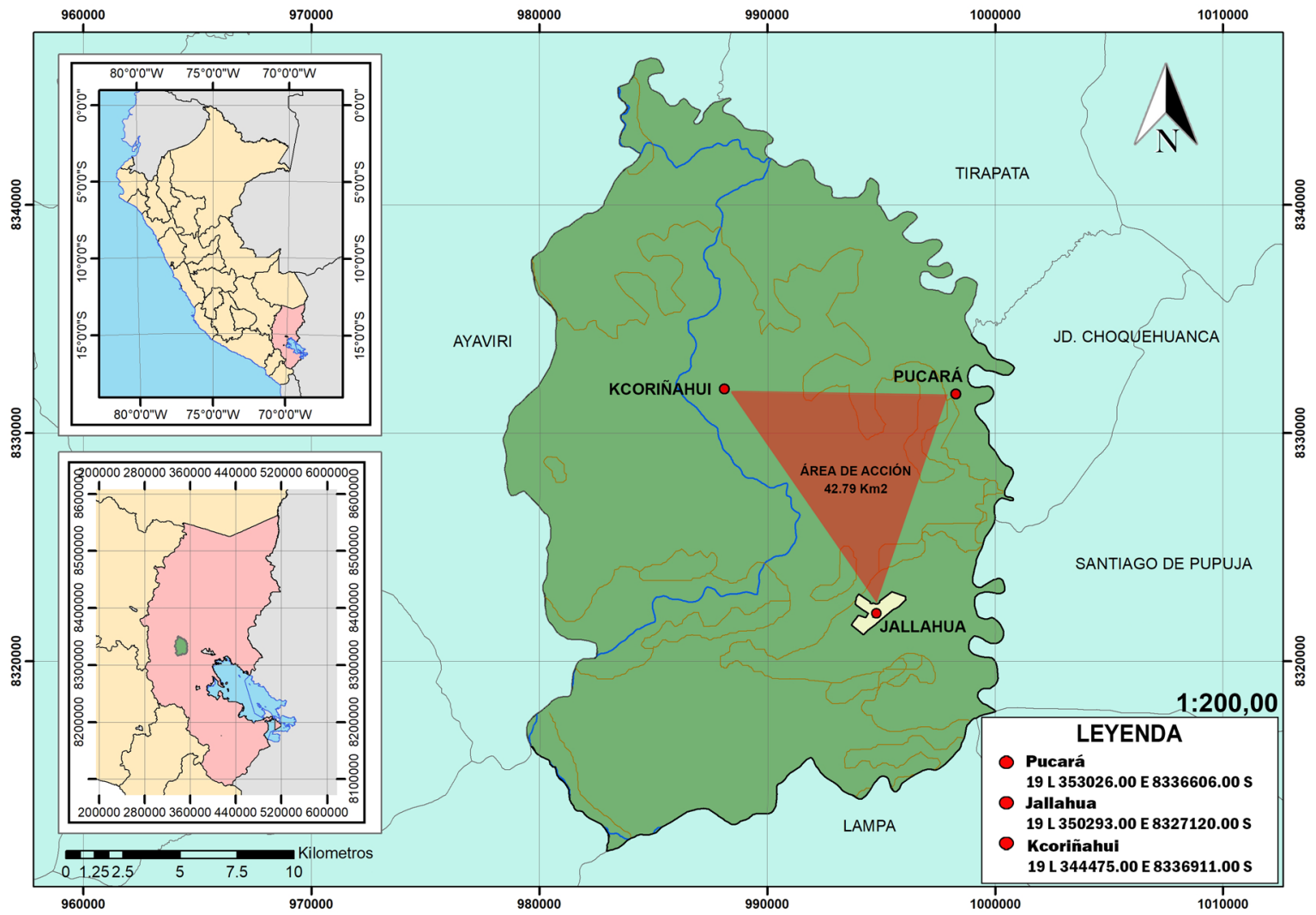

Figura 1. Detalle de la ubicación de las comunidades donde se realizó la colecta de especímenes de gorgojos de los Andes (Pucará-Lampa-Puno)
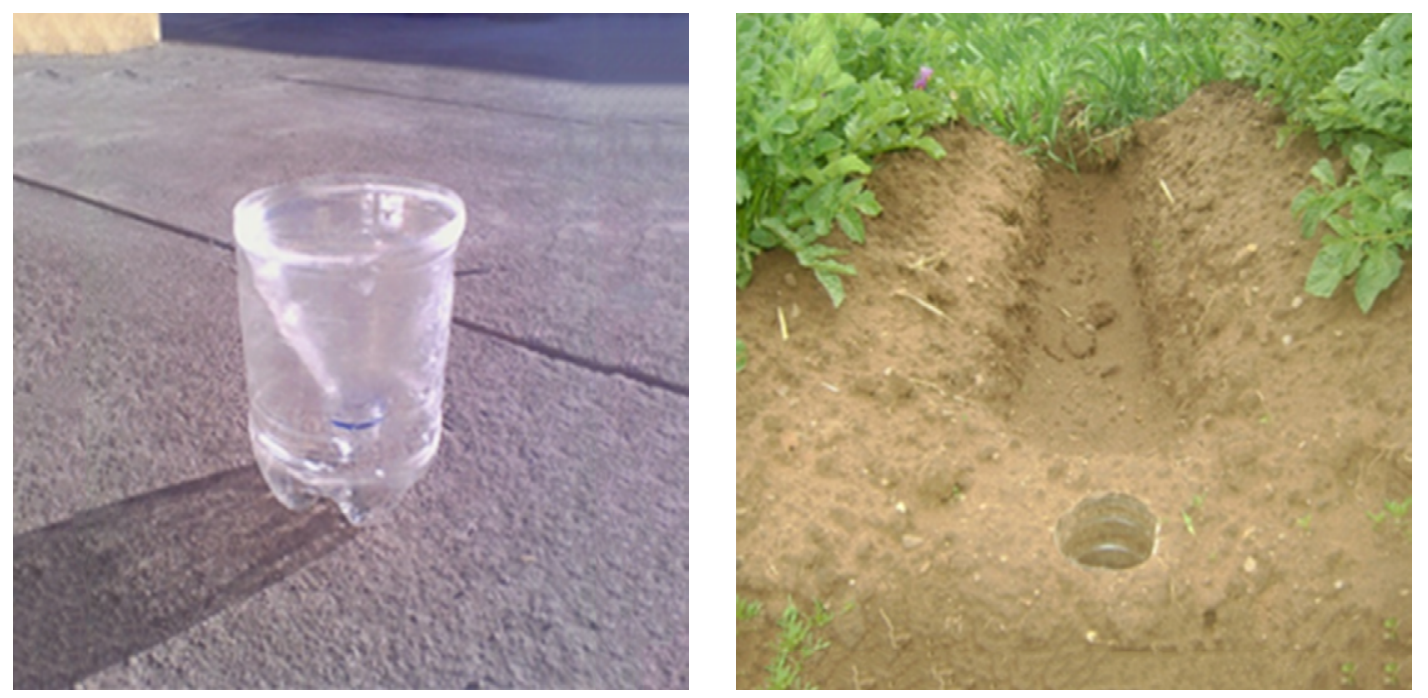

Figura 2. A. Trampa de caída modificada. B. Trampa enterrada con apertura a nivel del suelo

\section{Resultados}

Identificación de especies de gorgojos de los Andes en dos comunidades campesinas. Como resultado de las colectas de adultos de gorgojos de los Andes en las comunidades Jallahua y Kcoriñahui Pucará (Lampa-Puno), se identificaron las siguientes especies: Cylydrorhinus sp., Listroderes punicola Kuschel, 1949, Premnotrypes solaniperda Kuschel, 1956, Puranius sp., Rhigopsidius tucumanus Heller, 1906, Rhigopsidius piercei Heller, 1936 y cuatro morfotipos o morfoespecies aún no identificadas taxonómicamente.
Premnotrypes solaniperda Kuschel, 1956 (Fig. 3 A-B).

Según, la clave de Kuschel (1956), P. solaniperda se puede identificar por la combinación de las siguientes características morfológicas: Escapo de las antenas no muy grueso, sobrepasando ligeramente el margen anterior de los ojos, abultamiento lateral del protórax sobresaliente muy notorio, élitros convexos ligeramente más anchos que el protórax y a partir de la quinta interestría caen verticalmente a los costados; predominantemente en la parte alta del declive de los élitros presentan seis tubérculos pronunciados claramente visibles, cuatro de ellos de mayor tamaño y dos centrales de menor 
tamaño formando claramente un hexágono. Las hembras miden en promedio $4,5 \mathrm{~mm}$ de ancho y $8 \mathrm{~mm}$ de largo, los machos 3,5 de ancho y 6,5 de largo.

\section{Cylydrorhinus sp. (Figs. 3 C-D).}

El escapo de las antenas llega hasta el margen posterior de los ojos, a simple vista no presentan tubérculos, salvo pequeñas prominencias a los costados de los élitros; en cambio tienen punteaduras alineadas longitudinalmente, con escamas redondeadas y setas de colores cobrizo y verdosas, tanto a los costados del protórax como cubriendo los élitros; tibias medias con un solo espolón, en cambio las posteriores con dos espolones, fémures con escamas negras y verdosas, tibias considerablemente más largas que el tarso; los especímenes miden en promedio $3 \mathrm{~mm}$ de ancho y 5,5 $\mathrm{mm}$ de largo.

\section{Listroderes punicola Kuschel, 1949 (Fig. 3 E-F).}

Según, la clave de Kuschel (1949), L. punicola se puede identificar por la combinación de las siguientes características morfológicas: Son de un color marrón oscuro, excepto las antenas y los tarsos que son de color cobrizo, escamas redondeadas que cubren el protórax en el que se nota claramente una línea central y débilmente una línea en cada borde lateral; los élitros con escamas uniformemente distribuidas, algunas de ellas blanquecinas y otras cobrizas; en el declive de los élitros presentan un par de tubérculos cónicos y en la parte final de los élitros escamas de colores negro y blanco, las cuales se distribuyen en forma de "V". Las patas con abundante pubescencia; en promedio miden $3 \mathrm{~mm}$ de ancho y $8,5 \mathrm{~mm}$ de largo.

\section{Puranius sp. (Fig. 3 G-H).}

$\mathrm{Su}$ conformación es ligeramente parecida a Premnotrypes solaniperda, pero se diferencia por su menor tamaño y la presencia de tubérculos con setas a manera de grupos de espinas, mayormente de color marrón. Las antenas con clava gruesa; el protórax con tubérculos más o menos definidos; en los élitros los tubérculos se encuentran alineados con el eje longitudinal del cuerpo, la mayoría de ellos son del mismo tamaño y con las setas curvadas, a manera de espinas; intervalos elitrales planos. Los adultos miden 2,6 $\mathrm{mm}$ de ancho y 4,5 $\mathrm{mm}$ de largo; a pesar de ser pequeños, por la presencia de setas en los tubérculos aparentan tener una contextura robusta.

\section{Rhigopsidius tucumanus Heller, 1906 (Fig. 3 I-J).}

Según la descripción en las claves de Morrone \& Loiacono (1992), R. tucumanus se puede identificar por la combinación de las siguientes características morfológicas: Presentan el tegumento de color marrón oscuro, los ojos sub triangulares, protórax ligeramente más ancho que largo, con quillas laterales que dan la apariencia de márgenes afilados, en la parte posterior central pequeños tubérculos y a los costados tubérculos alargados más grandes; los élitros dorsalmente convexos, con escamas redondeadas, setas distribuidas irregularmente en todo el cuerpo y tubérculos irregulares poco sobresalientes, excepto cuatro tubérculos notorios antes del declive de los élitros; lateralmente los tubérculos están dispuestos en tres hileras a manera de una carena o pliegues curvilíneos, margen posterior uniformemente curvado; en promedio miden $3 \mathrm{~mm}$ de ancho y $6,5 \mathrm{~mm}$ de largo.
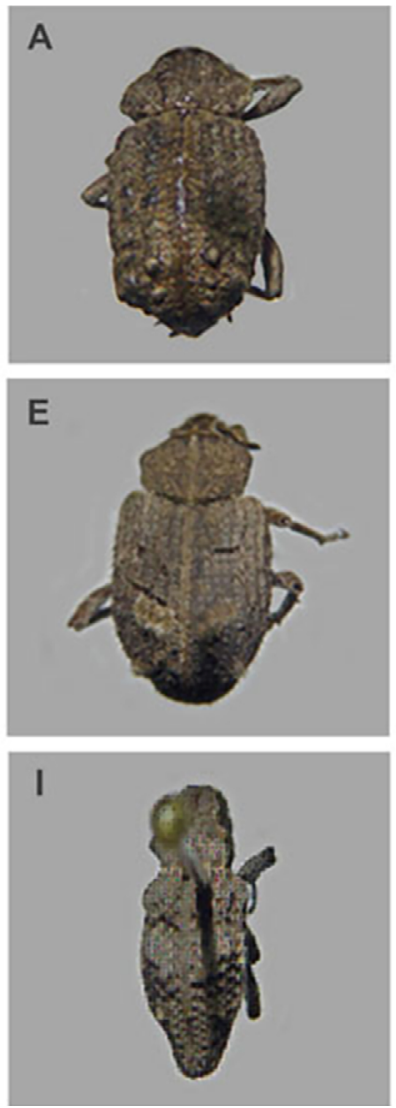
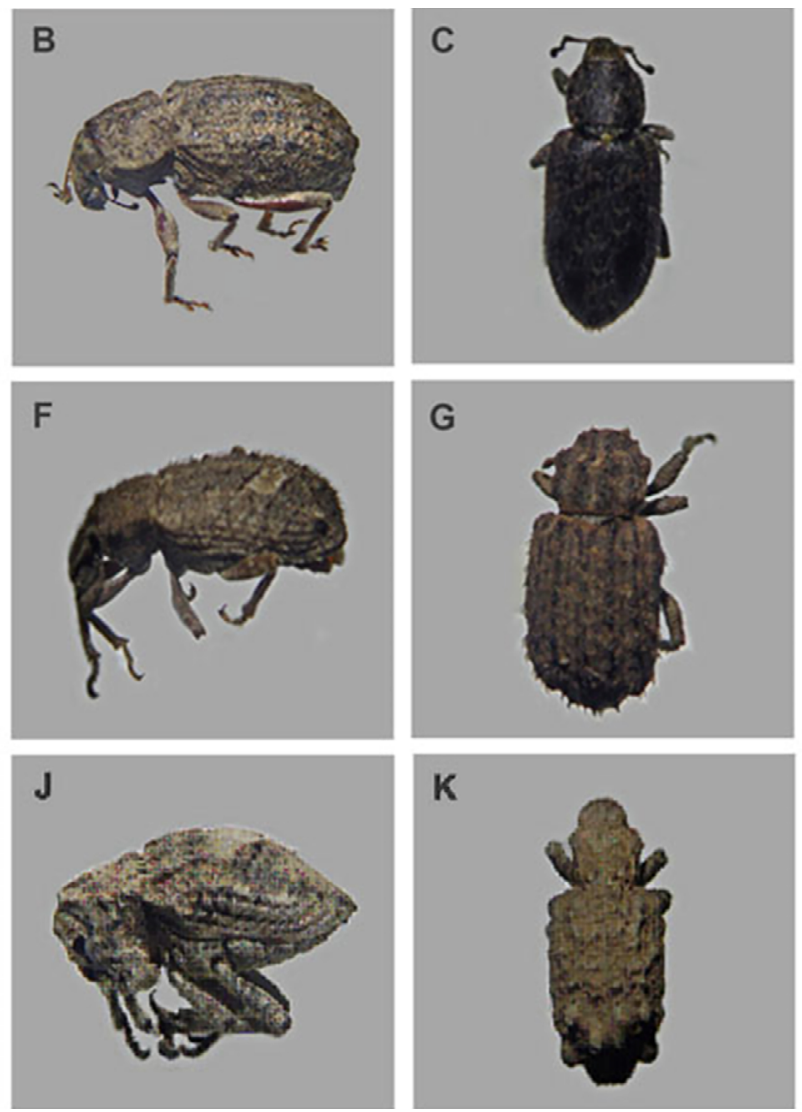
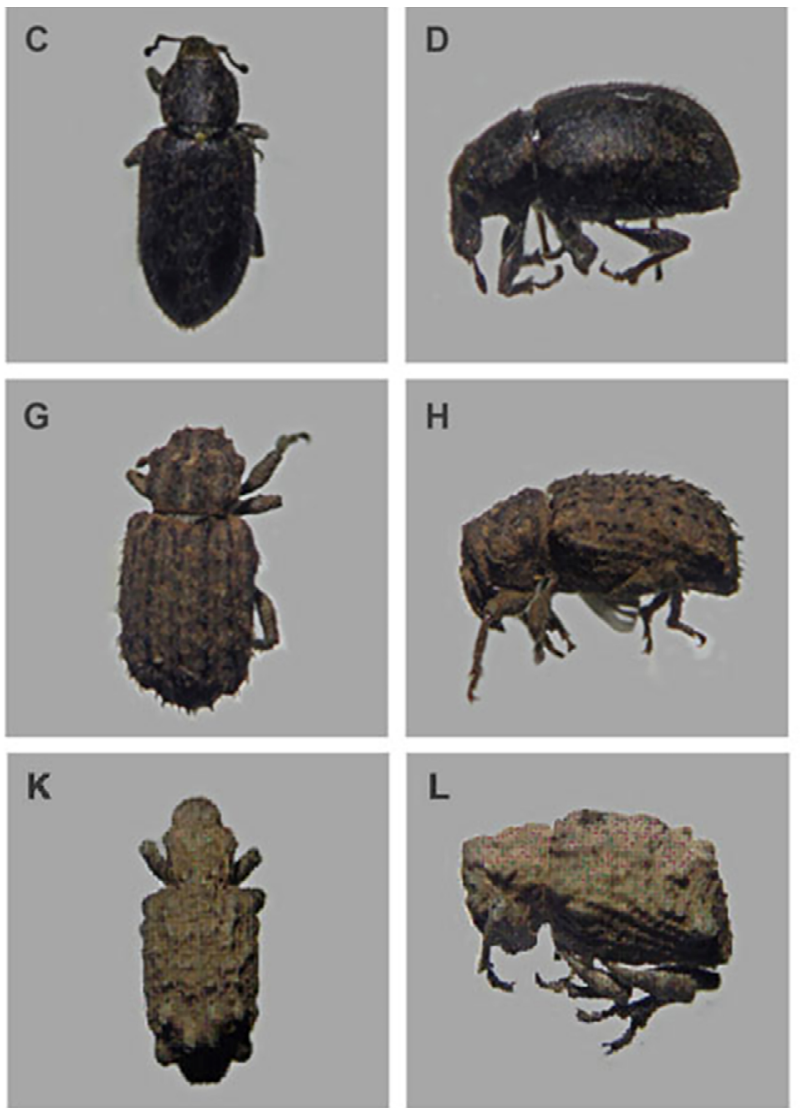

Figura 3. A. Vista dorsal. B. Vista lateral de Premnotrypes solaniperda Kuschel. C. Vista dorsal. D. Vista lateral de Cylydrorhinus sp. E. Vista dorsal. F. Vista lateral de Listroderes punicola Kuschel. G. Vista dorsal. H. Vista lateral de Puranius sp. I. Vista dorsal. J. Vista lateral de Rigopsidius tucumanus Heller. K. Vista dorsal. L. Vista lateral de Rhigopsidius piercei Heller (Pucará -Puno). 


\section{Rhigopsidius piercei Heller, 1936 (Figs. 3 K-L).}

Según las características de la clave presentada por Morrone \& Loiacono (1992), R. piercei se puede identificar, por la combinación de las siguientes características morfológicas: Rostro más alargado que el de $R$. tucumanus con tubérculos que son protuberancias en forma de dedos; los márgenes del protórax gruesos y dentados, a diferencia de $R$. tucumanus que son afilados; tubérculos grandes e irregulares distribuidos dorsalmente tanto en el protórax como en los élitros, los cuatro de mayor tamaño se ubican en la parte del declive elitral y son fuertemente ovalados, diferente a $R$. tucumanus que tiene los élitros convexos con tubérculos poco sobresalientes; lateralmente los tubérculos se encuentran distribuidos en tres hileras rectas, en cambio en $R$. tucumanus las hileras son curvilíneas $\mathrm{y}$ el márgen posterior es rectangular y dentado, mientras que en $R$. piercei, el márgen posterior es ligeramente curvado; presentan setas distribuidas indistintamente en todo el cuerpo y miden en promedio $4 \mathrm{~mm}$ de ancho y $7 \mathrm{~mm}$ de largo.

Además de las especies identificadas taxonómicamente, se diferenciaron cuatro morfotipos o morfoespecies, aún no identificados para las que se brindan algunas características morfológicas en la Tabla 1 y Fig. 4A-H.
Densidad poblacional y diversidad de especies. Las especies identificadas y los morfotipos no identificados se encuentran distribuidos heterogéneamente en las comunidades muestreadas, a altitudes entre 3883 y $4093 \mathrm{msnm}$. Varias especies de gorgojos de los Andes coexisten en el mismo hábitat, debido a que el alimento es abundante, siendo interesante hacer notar, que hay especies que estando presentes en una de las comunidades no se registran en la otra (Tabla 2) lo cual indica la heterogeneidad en la distribución geográfica.

La medida de diversidad considerada más adecuada, puesto que pondera a todas las especies según su importancia relativa, es la exponencial del índice de Shannon (Jost y Gonzales-Oreja 2012). Precisa que a menor índice mayor dominancia, lo cual surge con claridad en los resultados encontrados en el presente trabajo (Tabla 2), con índices de diversidad que se expresan en función de los índices de dominancia, que son de $-1,35$ y $-0,81$ para Premnotrypes solaniperda, siendo la especie más dominante, seguida por el morfotipo 2 en Jallahua y el morfotipo 3 en Kcoriñahui con índices de 0,98 y 0,95 , respectivamente. La fluctuación poblacional de la especie dominante $P$. solaniperda, en las dos comunidades se muestra en la (Figura 5).

Tabla 1. Características de morfotipos (no identificados) de "gorgojo de los Andes" Pucará (Lampa-Puno)

\begin{tabular}{cccc}
\hline Especie & $\begin{array}{c}\text { Largo } \\
(\mathbf{m m})\end{array}$ & $\begin{array}{c}\text { Ancho } \\
(\mathbf{m m})\end{array}$ & Características \\
\hline $\begin{array}{c}\text { Morfotipo 1 } \\
\text { (Fig. 4 A-B) }\end{array}$ & 6 & 2,5 & $\begin{array}{l}\text { Ëlitros color marrón, sin tubérculos, con manchas blancas paralelas al eje central } \\
\text { y con una mancha clara en forma de V en la parte posterior }\end{array}$ \\
\hline $\begin{array}{l}\text { Morfotipo 2 } \\
\text { (Fig. 4 C-D) }\end{array}$ & 7 & 3 & $\begin{array}{l}\text { Gris oscuro con manchas blanquecino-amarillentas en los élitros, tubérculos } \\
\text { distribuidos regularmente y cuatro más pronunciados en el declive elitral. }\end{array}$ \\
\hline $\begin{array}{l}\text { Morfotipo 3 } \\
\text { (Fig. 4 E-F) }\end{array}$ & 6 & 2,6 & Color negro en todo el cuerpo, bastante pubescente, no presenta tubérculos \\
\hline $\begin{array}{l}\text { Morfotipo 4 } \\
\text { (Fig. 4 G-H) }\end{array}$ & 8 & 3 & $\begin{array}{l}\text { Color marrón claro con bordes amarillo-verdoso, sin tubérculos y con bastante } \\
\text { pubescencia. }\end{array}$ \\
\hline
\end{tabular}

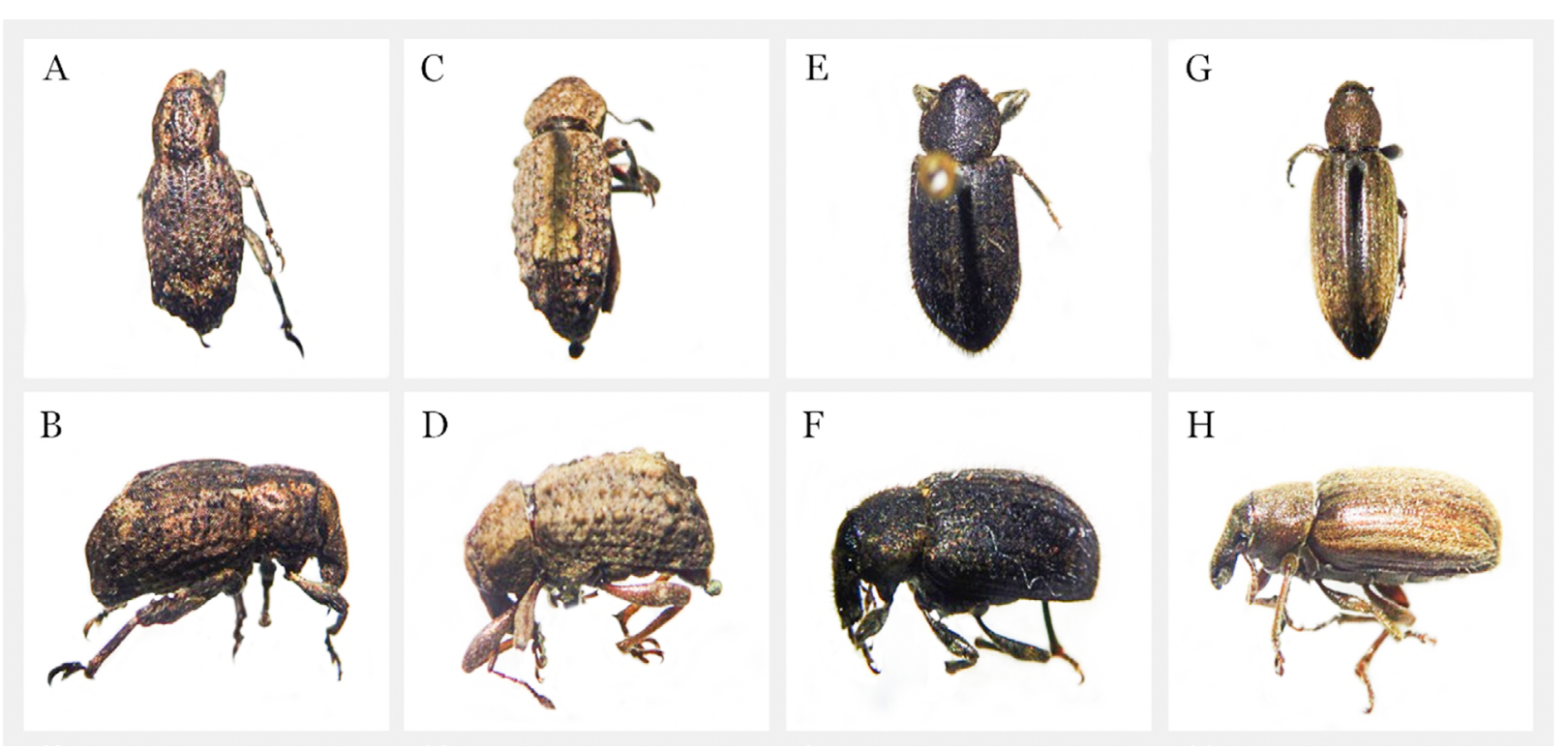

Figura 4. A. Vista dorsal. B. Vista lateral de morfotipo 1. C. Vista dorsal. D. Vista lateral de morfotipo 2. E. Vista dorsal. F. Vista lateral de morfotipo 3 . G. Vista dorsal. H. Vista lateral de morfotipo 4 (Pucará -Puno). 
Tabla 2. Densidad poblacional de especies e índice de diversidad en las comunidades Jallahua y Kcoriñahui en Pucara (Lampa-Puno).

\begin{tabular}{l|ccc|ccc}
\hline & \multicolumn{2}{|c|}{ Comunidad Jallahua (3.883 msnm) } & \multicolumn{3}{c}{ Comunidad Koriñahui (4.093 msnm) } \\
\hline \multicolumn{1}{c|}{ Especies capturadas } & $\begin{array}{c}\text { Densidad } \\
\text { Poblacional }\end{array}$ & $\begin{array}{c}\text { \% de } \\
\text { población }\end{array}$ & $\begin{array}{c}\text { Índice de } \\
\text { diversidad }\end{array}$ & $\begin{array}{c}\text { Densidad } \\
\text { poblacional }\end{array}$ & $\begin{array}{c}\text { \% de } \\
\text { Población }\end{array}$ & $\begin{array}{c}\text { Índice de * } \\
\text { diversidad }\end{array}$ \\
\hline Premnotrypes solaniperda & 930 & 88,6 & $-1,35$ & 104 & 77,6 & $-0,81$ \\
\hline Cylydrorhinus sp. & 0 & 0 & 0 & 4 & 2,99 & 0,99 \\
\hline Puranius sp. & 5 & 0,50 & 1,00 & 1 & 0,75 & 1,00 \\
\hline Listroderes punicola & 2 & 0,19 & 1,00 & 1 & 0,75 & 1,00 \\
\hline Rigopsidius tucumanus & 0 & 0 & 0 & 1 & 0,75 & 1.00 \\
\hline Rigopsidius piercei & 1 & 0,10 & 1,00 & 0 & 0 & 0 \\
\hline Morfotipo 1 & 0 & 0 & 0 & 5 & 3,73 & 0,99 \\
\hline Morfotipo 2 & 84 & 8,00 & 0,98 & 0 & 0 & 0 \\
\hline Morfotipo 3 & 25 & 2,38 & 0,99 & 17 & 12,68 & 0,95 \\
\hline Morfotipo 4 & 3 & 0,29 & 1,00 & 1 & $\mathbf{1 3 4}$ & $\mathbf{1 0 0}$ \\
\hline Total individuos capturados & $\mathbf{1 0 5 0}$ & $\mathbf{1 0 0}$ & & 0,75 & 1,00 \\
\hline
\end{tabular}

(*) Índice de diversidad = dominancia: a menor índice mayor dominancia (Jost y Gonzales-Oreja, 2012)

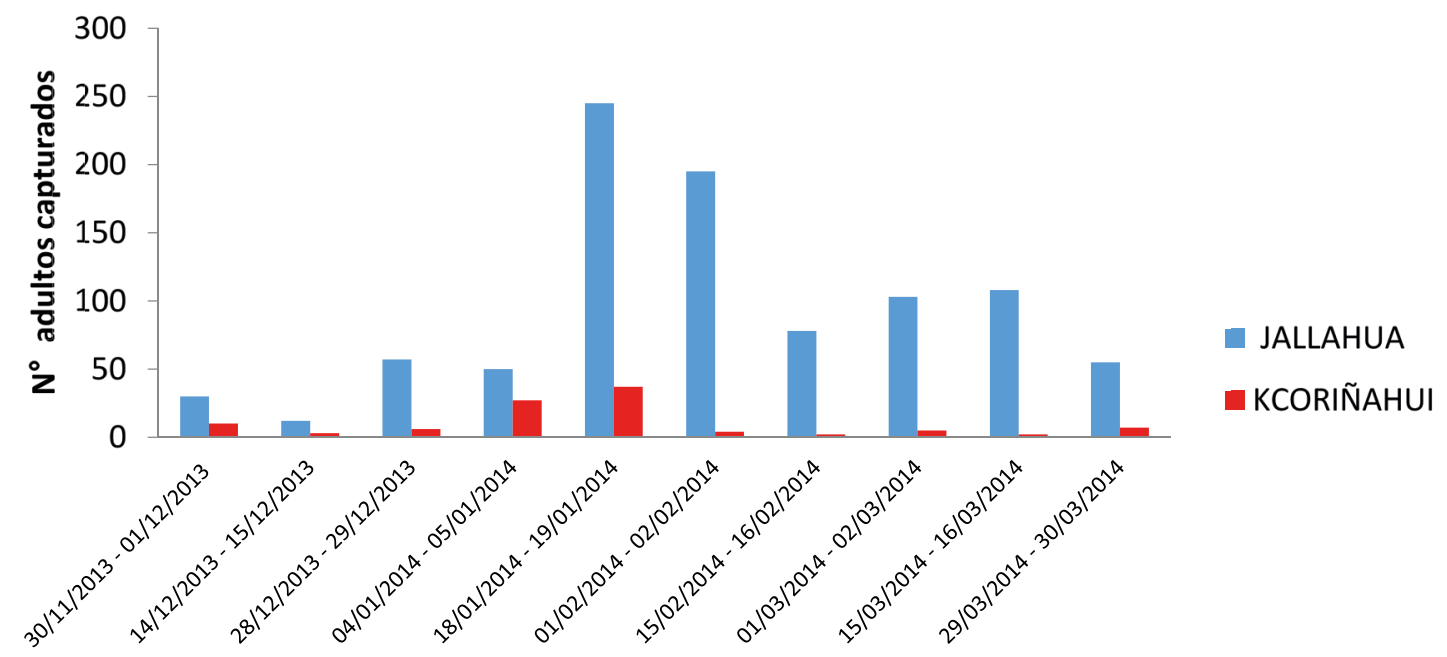

Fechas de evaluación

Figura 5. Fluctuación poblacional de Premnotrypes solaniperda (especie dominante).

\section{Discusión}

Identificación de especies de gorgojo de los Andes. En Puno el cultivo de papa es el de mayor importancia económica y social, encontrándose variedades dulces, amargas, nativas y mejoradas, las cuales tienen como principales enemigos a diversas especies del complejo gorgojo de los Andes. Esto contrasta con otros lugares productores de papa a lo largo de la cadena de los Andes latinoamericanos, que son afectados por una o pocas especies del complejo de gorgojos, de acuerdo con información aportada por Cahuana y Arcos (2002), Alcázar (2002, 2016), Bravo (2010), Zapana (2015), y Loza y Bravo (2015).

La identificación con base en características morfológicas de las especies de gorgojo de los Andes colectadas en las comunidades muestreadas, no ofrece dificultades al usar las claves dicotómicas y trabajos taxonómicos, preparados por especialistas y autores de las especies descritas (; Kuschel 1949, 1956; Morrone y Loiácono 1992; Barea et al. 2016; Yábar-Landa et al. 2011; Kroschel et al. 2012).

En el caso de Cylydrorhinus sp. se trata de una especie, no citada anteriormente en el altiplano, con características morfológicas diferentes a las del género Premnotrypes, que carece de tubérculos y tiene escamas de colores cobrizos, verdosas y negras sobre los élitros tal como lo señala en sus claves y descripción Kuschel (1956), características que coinciden ampliamente con los escasos ejemplares colectados en la comunidad Koriñahui a una altitud de 4093 msnm, en áreas de mezclas dulces y amargas de papa.

Entre las especies colectadas, no se encontró ninguna que concuerde con las características morfológicas de Premnotrypes pusilus, citada por Yábar-Landa (2015b) y reportada para altitudes de 3690 a 4000 msnm en las zonas de Apurímac, Cusco, Junín y Pasco. Se asume que posiblemente se 
encuentre en otros lugares del altiplano de Puno. Tampoco se registraron especies que tengan las características de los géneros Rhinotrypes y Microtrypes, que Yábar-Landa (2019) mencionaron para Perú y Bolivia; sin embargo, el género Microtrypes ha sido mencionado por Bravo (2010) como plaga en tubérculos de oca Oxalis tuberosa.

Se coincide con Kuschel (1956) acerca de que los Premnotrypini se encuentran por lo general sobre los $3000 \mathrm{msnm}$. También con Yábar-Landa (1994), quien indicó que la mayoría de las especies del complejo de gorgojos de los Andes se hallan adaptadas a altitudes que van desde 2100 a $4350 \mathrm{msnm}$.

Densidad poblacional y diversidad de especies. Las densidades poblacionales de las especies registradas indican que $P$. solaniperda es la más abundante en las dos comunidades estudiadas. Esta especie incrementa paulatinamente la población de adultos desde el mes de noviembre, hasta alcanzar el pico más alto en enero para luego descender hasta fines de marzo (Figura 5). Lo anterior coincide con otros autores como Bravo (2010), Yábar-Landa (2011), y Kroschel (2012).

Se considera que la variable más importante sería la condición de humedad del suelo. Los morfotipos 2 y 3 le siguen en abundancia y el resto de las especies muestran densidades poblacionales entre 1 y 5 individuos; coincidiendo con lo señalado por Begón y Townsend (1995), Cabello et al. (1999), Lante et al. (2013), Yábar (2015a) y Guzmán et al. (2016). Además, las condiciones climáticas, edáficas y las prácticas agronómicas tendrían gran influencia en la densidad y distribución de las especies, ratificando los conceptos de Krebs (1985).

\section{Conclusiones}

En el altiplano peruano existe una alta diversidad de especies del complejo de gorgojo de los Andes; de 10 especies colectadas, seis fueron identificadas taxonómicamente a nivel de especie, con evidente dominancia de $P$. solaniperda, en ambas comunidades estudiadas en Puno.

La especie Cylydrorhinus sp. es la única citada por primera vez para esta parte del altiplano peruano.

\section{Agradecimientos}

Los autores expresan su agradecimiento por el apoyo y contribución al Ing. M.Sc. Jesús Alcázar Sedano en la identificación por comparación de las especies de gorgojo de los Andes, con los ejemplares de colección de referencia del museo del Centro Internacional de la papa CIP, así como por sus sugerencias para la mejor conducción de la investigación en campo.

\section{Literatura citada}

ALCÁZAR, J. 2002. Biología del gorgojo de los Andes Premnotrypes spp. (Coleoptera: Curculionidae). Manual de capacitación, fascículo 1. Centro Internacional de la Papa. $13 \mathrm{p}$.

ALCÁZAR, J.; SALAS, A.; NAVARRETE, V.; KROSCHEL, J.; ROCA, W.; ELLIS, D.; VEGA, A.; POMA, E.; QUISPE, V. 2016. Evaluación de la susceptibilidad de genotipos de especies silvestres de papa (Solanum spp.) al gorgojo de los Andes Premnotrypes suturicallus Kuschel. En: Huanca Maldonado, J,; Alcázar Sedano, J. (eds). LVII Convención Nacional de Entomología. Resúmenes. Sociedad Entomológica del Perú; Universidad Nacional Agraria La Molina. . Lima. Perú. 2 p. https:/hdl.handle.net/10568/96100
BAREA, O.; ANDREW, R. 1997. Biología, daño y control químico del gorgojo de los Andes, Rhigopsidius tucumanus Heller. Revista Latinoamericana de la Papa, 9 (10): 96-105. https://doi. org/10.37066/ralap.v9i1.83

BEGÓN M.; HARPER, J.; TOWNSEND, C. 1995. Ecología, individuos, poblaciones y comunidades. Editorial Blackwell Scientific Publications. Oxford. Reino Unido. 876 p.

BRAVO, R. 2010. Manejo Agroecológico de Plagas Andinas. Libro. Primera edición. Editorial el Altiplano. E.I.R.L. Puno, Perú. $131 \mathrm{p}$.

CABEllo, J.; ALEMÁN, M.; CASTRO, H.; MOTA, J. 1999. Diversidad ecológica en comunidades de matorral mediterráneas-semiáridas del sureste de la península Ibérica. Departamento de Biología Vegetal y Ecología, Universidad de Almería. España.

CAHUANA, R.; ARCOS, J. 2002. Variedades nativas y mejoradas de papa en Puno. Serie libro-01. Edit. Estación Experimental Illpa-INIA. 120 p. https://repositorio.inia.gob.pe/ handle/20.500.12955/898

GIORDANENGO, P.; VINCENT, C.; ALYOKHIN, A. 2013. Insect pests of potato. Global Perspectives on Biology and Management. En: Philippe Giordenango, Charles Vincent y Andrei Alyokhin (Eds.). Insect Pests of Potato . Academic Press is an imprint of Elsevier. The Boulevard, Langford Lane, Kidlington, Oxford USA. 598 p. https://doi.org/10.1016/C2010-0-67998-3

GUZMÁN-MENDOZA， R.; CALZONTZI-MARÍN, J.; SALAS-ARAIZA, M. D.; MARTÍNEZ-YÁÑEZ, R. 2016. La riqueza biológica de los insectos: análisis de su importancia multidimensional. Ensayo. Acta Zoológica Mexicana 32 (3): 370379. http://www.scielo.org.mx/scielo.php?script=sci_arttext\& pid=S0065-17372016000300370

JARANDILLA. R. C. 2010. Estudio de la dinámica poblacional de especímenes del gorgojo de los Andes (Premnotrypes sp. y Rhigopsidius piercei) en el Altiplano Central Provincia Aroma. Tesis Ingeniero Agrónomo. Universidad Mayor San Andrés. La Paz. Bolivia. 101 p. https://repositorio.umsa.bo/browse? type $=$ author \&value $=$ Jarandilla + Rodriguez $\% 2 C+$ Claudia

JOST, L.; GONZÁLEZ-OREJA, J. 2012. Midiendo la diversidad biológica: más allá del índice de Shannon. Comentario. Acta Zoológica Lilloana 56 (1-2): 3-14. http://www.lillo.org.ar/jour nals/index.php/acta-zoologica-lilloana/article/view/240

KUSCHEL, G. 1949. Los "Curculionidae" del Extremo Norte de Chile. Acta Zoológica Lilloana. Tomo VIII. Universidad Nacional de Tucumán. Instituto: Miguel Lillo. Tucumán Republican Argentina. 5- 54. http://weevil.info/los-curculionidae-del-extremo-norte-de-chile-coleoptera-curcul-ap-6

KUSCHEL, G. 1956. Revisión de los Premnotrypini y adiciones a los Bagoini. Boletín Museo Nacional de Historia Natural (Chile) 26: 187-235. https://publicaciones.mnhn.gob.cl/668/articles-63959_ archivo_01.pdf

KREBS, C. H. 1985. Ecología: estudio de la distribución y la abundancia. Editorial. Libro. Harla, S.A. México. 753 p.

KROSCHEL, J.; CAÑEDO, V.; ALCÁZAR, J.; MIETHBAUER, T. 2012. Manejo de plagas de la papa en la región andina del Perú. Guía de capacitación Segunda edición. Centro Internacional de la Papa, Lima, Perú. 88 p. https://hdl.handle.net/10568/96080

LOZA, A.; BRAVO, R.; DELGADO, P. 2015. Refugios artificiales para comunidades de artrópodos depredadores epígeos y su efecto en el control biológico del gorgojo de los Andes en el cultivo de papa, Puno - Perú. Revista Peruana de Entomología 50 (2): 13-25. https://repositorio.inia.gob.pe/bitstream/20.500.12955/824/1/ Loza-Refugios_artificiales_artr\% $\% 3 \%$ B3podos.pdf

MORENO, C. E. 2002. Métodos para medir la biodiversidad. Recensión de libro. Acta Zoológica Mexicana 85: 195-196. http://www.scielo.org.mx/scielo.php?script=sci_arttext\& pid=S0065-17372002000100016

MORRONE, J.; LOIACONO, M. 1992. Revision of the genus Rhygopsidius Heller (Insecta Coleoptera, Curculionidae: Rhytitthininae). 
Entomologische Abhandlungen. Statliches Museum für Tierkunde Dresden 54 (7): 129-139.

YÁBAR, E. 1994. Manejo Ecológico del gorgojo de los Andes. Ediciones Red de Acción en Alternativas al uso de Agroquímicos (RAAA). Perú. 119 p.

YÁBAR-LANDA, E.; GARCIA, H.; SAAVEDRA, J.; DE LOS RÍOS, H. 2011. Curculionidos (Coleoptera: Curculionidae) asociados al tubérculo de la papa bajo condiciones de almacenamiento tradicional en Cuzco, Perú. Boletín del Museo de Entomología de la Universidad del Valle 12 (1): 42-47. https://drive.google.com/file/d/1CDuc8YvLuskGEI_S4H_ bqOnu78W_A4mW/view

YÁBAR-LANDA, E. 2015a. Biodiversidad y cambio climático: Diversidad de insectos en cuatro ecotipos de papa. Climate Change in the Tropical Andes 3 1-5. http://revistas.unsaac.edu.pe/index. $\mathrm{php} / \mathrm{rccat} / \mathrm{article} / \mathrm{view} / 350$

YÁBAR-LANDA, E. 2015b. Re descripción de Premnotrypes pusillus Kuschel (Coleoptera, Curculionidae, Entiminae) y ampliación de su distribución conocida en Perú. Boletín de la Sociedad Entomológica Aragonesa (SEA) 56 (30/06/2015): 375-377.http://www.sea-socios.com/Boletines/PDF/Boletin56/375-377BSEA56NB_Premnotrypes_pusillus_Peru.pdf

YÁBAR-LANDA. E. 2019. Nuevos registros de Microtrypes Kuschel y nuevas localidades para Rhinotrypes Kuschel (Coleoptera, Curculionidae, Entiminae) en Perú, Con una clave de identificación. Revista Peruana de Biología 26 (2): 183-188. https://doi.org/10.15381/rpb.v26i2.15095

ZAPANA-PARI, J.; VILLALTA, R. P.; ZAPANA, L. J.; ESCOBAR, M. F. 2015. Evaluación de substratos de Beauveria brongniartii para control de (Premnotrypes spp.) en cultivo de papa. Revista de Investigaciones Altoandinas 17 (3): 347-354. http://revistas. unap.edu.pe/rianew/index.php/ria/article/view/146 http://dx.doi. org/10.18271/ria.2015.146.

\section{Contribuciones de los autores}

Rosario Y. Bravo: Conceptualización, análisis formal, investigación, conservación de datos, metodología, supervisión, visualización, validación, redacción-borrador original, redacción-revisión y edición; Víctor Hugo Idme: Conceptualización, investigación, conservación de datos, metodología, redacción-borrador original, visualización, validación; Israel Lima: Análisis formal, software, visualización, validación, redacción-revisión y edición; Grover Cornejo: Metodología, software, visualización, redacción-revisión y edición; Víctor Hugo Casa: Análisis formal, metodología, software, visualización, validación, redacción-revisión y edición.

\section{Conflictos de interés}

Los autores declaramos no tener conflictos de intereses. 